\title{
Fiber-Optic Sensing System: Overview, Development and Deployment in Flight at NASA
}

\author{
Hon Man Chan, Allen R. Parker, Anthony Piazza, and W. Lance Richards \\ NASA Armstrong Flight Research Center \\ P.O. Box 273 \\ Edwards, California 93523 \\ hon.chan@nasa.gov
}

\begin{abstract}
An overview of the research and technological development of the fiber-optic sensing system (FOSS) at the National Aeronautics and Space Administration Armstrong Flight Research Center (NASA AFRC) is presented. Theory behind fiber Bragg grating (FBG) sensors, as well as interrogation technique based on optical frequency domain reflectometry (OFDR) is discussed. Assessment and validation of FOSS as an accurate measurement tool for structural health monitoring is realized in the laboratory environment as well as large-scale flight deployment.
\end{abstract}

Keywords-fiber Bragg gratings; fiber sensing; optical frequency domain reflectometry; interrogator

\section{INTRODUCTION}

It is a vision for aerospace engineers to be able to monitor the entire aero-frame of a structure with a network of multiple sensors capable of monitoring state-of-stress in real time as well as quantifying airframe loads or calculating the remaining available airframe fatigue life. The fiber-optic sensing system (FOSS) has the potential to dramatically improve the design and performance of aerospace vehicles. The FOSS concept involves sensors capable of being distributed in vast networks analogous to the nervous system in the human body. Structural health parameters such as stress, structural instabilities, temperature distribution, and other engineering measurements can be monitored in real time using FOSS, offering an unparalleled amount of valid engineering data indicating system health, with a negligible weight penalty.

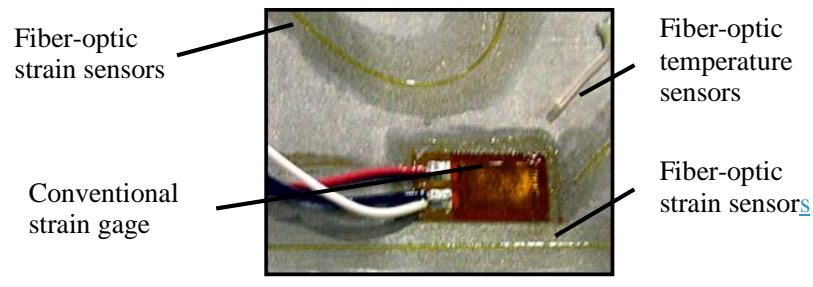

Fig. 1. Strain sensor size comparison.

In this conference paper, the merit of fiber optic sensors over the traditional strain gage is discussed first. An overview of fiber-sensor-based fiber Bragg gratings (FBG) operation, and how optical frequency domain reflectometry (OFDR) is utilized into interrogating thousands of multiplexing FBG is shown. Both ground-based and flight-based interrogation system are discussed. Sensing system validation is shown in controlled laboratory testing, as well as in large-scale NASA projects, in both ground test and flight test.

\section{BACKGROUND}

The foil strain gage has been the de facto industry standard for the past 70+ years for determining the state-of-stress of a structure [1]. Foil gages are advantageous because they are accurate, reliable, economical, and repeatable. Nevertheless, fiber-optics-based strain-sensing presents many advantages over conventional strain gages, especially regarding size and weight. Optical fibers, each approximately the diameter of a human hair, can be multiplexed thousands of sensors per fiber. Optical fibers are also chemically inert, and immune to both electromagnetic interference (EMI) and radiation. Due to their small size, optical fibers can be installed and embedded directly onto composite structure, or can be adhered to the surface of a structure, using the same method as with conventional strain gages. Unlike conventional strain gages, however, no soldering or clamping is required for fiber installation. Another weight advantage for fiber over conventional strain gages is that multiple sensors can be installed on a single fiber line, whereas a conventional single-axial strain gage requires three copper lead wires, as shown in Fig. 1, which can add substantial overall weight when hundreds of conventional strain gages are installed.

\section{THEORY}

Optical fiber sensors, composed of FBGs, are sensitive to both strain and temperature due to changes of the Bragg wavelength under environmental perturbation. Fiber Bragg gratings are created by exposing an optical fiber to an ultraviolet interference pattern, which produces a periodic change in the core index of refraction. These periodic changes lead to reflection of the unique Bragg wavelength $\left(\lambda_{B}\right)$, which is determined by the effective core index $\left(\mathrm{n}_{0}\right)$ as well as the modulation pitch size $(\Lambda)$ via (1) [2].

$$
\lambda_{B}=2 n_{0} \Lambda
$$


As a Bragg grating is stretched, the change of pitch size changes its Bragg wavelength. Assuming the effective index is constant, and the fiber is optically isotropic, by monitoring the change in Bragg wavelength, one can determine the strain change of the fiber grating via (2), where $\Delta \lambda_{B}$ is the change in Bragg wavelength, $\lambda_{B}$ is the unstrained Bragg wavelength, $p_{e}$ is the strain-optic coefficient, and $\varepsilon$ is the strain in the fiber direction.

$$
\varepsilon=\frac{1}{1-p_{e}} \frac{\Delta \lambda_{B}}{\lambda_{B}}
$$

A unique feature of FOSS over other fiber-optic interrogators is the ability to interrogate thousands of FBG sensors simultaneously on a single fiber. The FOSS system is based on method of OFDR to interrogate FBG [3]. Typical OFDR systems interpret a beat frequency between a reference arm and the sensing arm, where the device under test (DUT) is located, which form the interfering signal generated by the interferometer created from the optical network. The phase difference generated from the interferometer is then being detected by the photodetector.

This same interference effect is applied to FBGs used as strain sensors, shown in Fig. 2. In OFDR application of these sensors, the distance of each sensor can be individually determined first through sampling of output intensity while continuously sweeping the laser's wavelength. The output signal is a summation of the complete set of gratings located along the fiber length. Each grating is modulated with its unique frequency, determined by the grating's unique position on the fiber. These sensors are attached to an optical network for inspection.

$$
I_{R}=\sum_{i} R_{i} \operatorname{Cos}\left(k 2 n_{0} L_{i}\right) \quad k=\frac{2 \pi}{\lambda} \quad \begin{aligned}
& \mathrm{R}_{\mathrm{i}} \text { - spectrum of } \mathrm{i}^{\text {th }} \text { grating } \\
& \mathrm{n}_{0} \text { - effective index } \\
& \mathrm{L} \text { - path difference } \\
& \mathrm{k} \text { - wavenumber }
\end{aligned}
$$

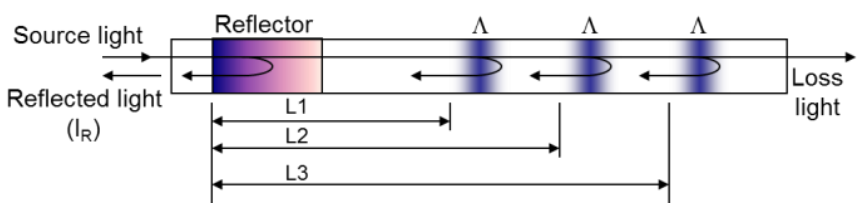

Fig. 2. Fiber with multiple Bragg gratings.

Applying a Fourier transform to the intensity information gathered from the wavelength sweep range, this information is then mapped from wavelength domain to spatial domain. From the spatial domain each corresponding reflection represents each grating at its unique distance. Since applied strain detected from each FBG is linear to its shift in its Bragg wavelength, each grating's wavelength can be determined by taking the inverse Fourier transform from the spatial domain back to the wavelength domain.

\section{INSTRUMENTATION}

\section{A. Fiber Optic Strain Calibration}

A typical optical fiber used is single-mode with a cladding diameter of $127 \mu \mathrm{m}$ and polyimide coating thickness of $7 \mu \mathrm{m}$. The optical fiber contains quasi-continuous FBGs, which enable system software to select spatial intervals from 0.25 inch and larger. Grating wavelengths are nominally written at $1550 \mathrm{~nm}$. For each lot of fiber received, a strain optic coefficient $\rho_{e}$ is derived prior to use. For this initial calibration, a cantilever beam is instrumented with foil strain gages and a short run of FBG optical fiber. Both types of sensors are connected to their respective data acquisition system. The $\rho_{e}$ calibration value is then adjusted up or down until the FBG indicated strain matches that of the strain gage as closely as practical.

\section{B. Fiber Optic Sensing System (FOSS)}

The FOSS developed at AFRC is used to interrogate the continuous FBG optical fiber and to provide strain and/or temperature data. It is capable of simultaneously and continuously interrogating up to eight 40 -foot optical fibers at 0.25 -inch spatial resolution for a total of 16,000 sensors per system. Although finer resolutions are possible, they are achieved at the cost of reduced fiber lengths. The systems can operate in stand-alone mode, which is used for flight applications, or remote control mode, in which a laptop is connected to provide monitoring and control. The maximum sample rate in flight is $100 \mathrm{~Hz}$, and a centralized software interface combines all functions into a suite of applications to fully exercise the FOSS.

\section{VALIDATION}

\section{A. Hollow Steel Beam}

In conjunction with the University of Mississippi, testing was conducted on two test articles: a hollow cantilevered steel beam and an ultralight carbon composite wing [4] with the objective of determining deflected wing shape and out-of-plane loads utilizing FBG sensors and FOSS technology. Fig. 3 shows the steel beam, which was instrumented with eight uniaxial strain gages located along the centerline of the lower surface. A single-mode fiber optic strand, consisting of 151 FBG sensors evenly distributed in 0.5 -inch increments, was mounted from the root to the tip of the beam along a centerline of the upper surface. The beam was 78.7-inches in length, 2.75-inches in diameter, and 0.12-inch thick. A universal test frame (UTF) was used as the support structure for testing.

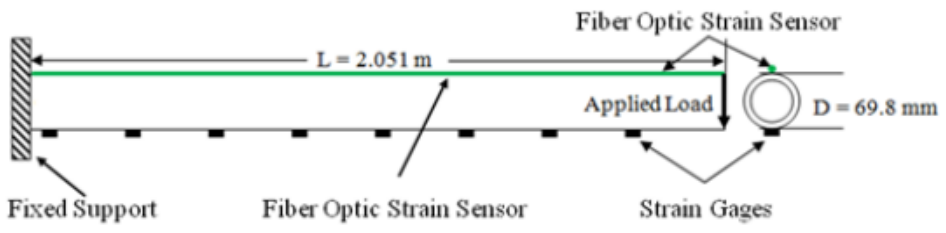

Fig. 3. Cantilevered steel beam instrumented with FBGs and strain gages, with cross-sectional view (right).

A tip load was applied to the free end of the beam by a hydraulic cylinder. To compare the measurements, the absolute value of the strain gage was plotted, in Fig. 4, with the analytical solution against the FBG strain data. Both the strain gage data are linear and have a correlation coefficient greater than 0.99 at all load levels and stations along the beam. At higher loads, however, the difference between the analytical 
solution and the measured data increased, due to the difficulty in achieving an idealized fixed support for the cantilevered beam.

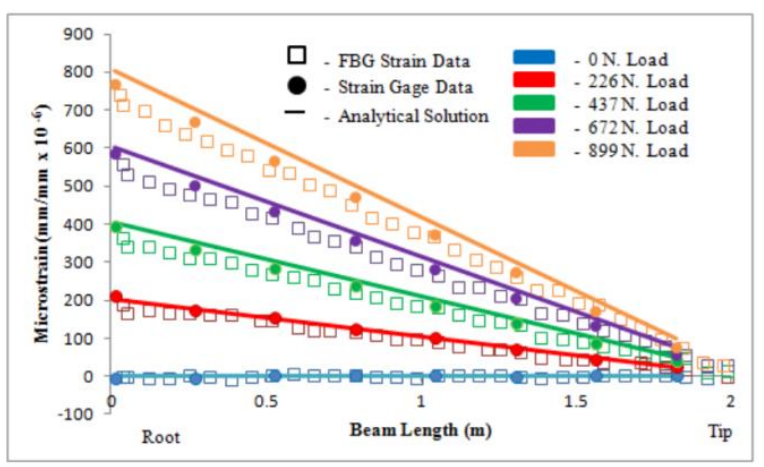

Fig. 4. FBG and conventional strain gage data subjected to tip loading.

\section{B. NASA Ikhana Flight Test}

In June 2003, the Helios prototype unmanned air vehicle (UAV) experienced significant pitch instability during low-altitude flight, which resulted in catastrophic structural failure, and the vehicle broke up in flight. Unfortunately, the use of conventional strain gage instrumentation was impractical due to the weight of the instrumentation. The viability of using FOSS as a wing shape sensor was considered.

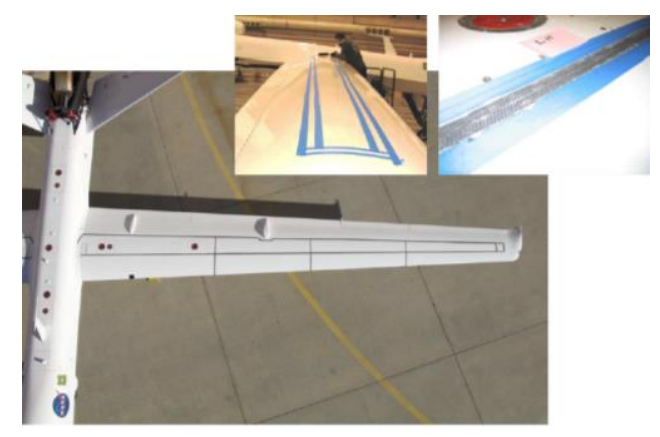

Fig. 5. Installation of FBG sensor on upper wing surface on the AFRC Predator-B UAV.

NASA AFRC acquired a Predator B unmanned science and technology development aircraft from General Atomics Aeronautical Systems Inc. in November 2006. The aircraft, named Ikhana, has a wingspan of 66 feet and is 36 feet in length. The vehicle has the capability of carrying 400 pounds of sensors and equipment internally and over 7,000 pounds of payload in the under-wing pods. The aircraft was designed to fly long-endurance missions at high altitudes (above 40,000 feet). The NASA AFRC-designed FOSS system was integrated into the avionics bay of the Ikhana vehicle.
Six 20-foot FBG optical fibers were affixed to the wings. The strain optic coefficient $\rho_{e}$ of the fiber was 0.79 and the spatial resolution of the sensors was $0.5 \mathrm{inch}$. In addition to the 2,880 FBG sensors, 16 strain gages were used to validate FOSS measurements. Fig. 5 illustrates the placement of the sensors installed for flight-testing. From May until August 2008, 18 flight tests totaling 36 flight hours were conducted on the Ikhana vehicle. As can be seen in Fig. 6, there is significant similarity in the data, which indicates the accuracy and validity of the optical fiber strain measurement. The strain data from individual FBG sensors closely compare to the strain gage measurements and have a correlation coefficient greater than 0.99 .

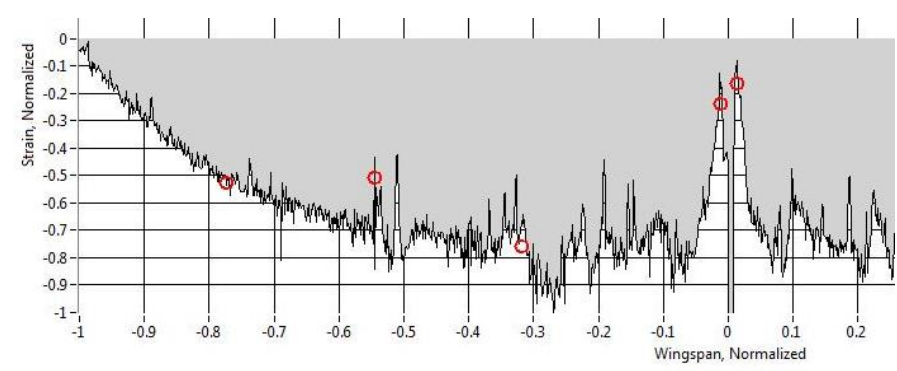

Fig. 6. Normalized strain data mapping across the wingspan of Ikhana (outlined) versus co-locating strain gage data (circular).

\section{CONCLUSION}

Fiber-optic sensing systems (FOSS) have the potential to dramatically improve the design and performance of aerospace vehicles. Fiber Bragg grating sensors are comparable to strain gage measurements in numerous real-world applications. An overview of the results of real-world testing performed by the National Aeronautics and Space Administration Armstrong Flight Research Center conclusively shows that FBG sensors are as accurate as traditional strain gages, and in addition provide advantages with regard to weight and size. The prospect of using FOSS in aerospace structural health monitoring, dramatically improving the measurement count to total sensor weight, has the potential to benefit vehicle management and operations from early design to final retirement.

\section{REFERENCES}

[1] Kreuzer, M., Strain Measurement with Fiber Bragg Grating Sensors, HBM, Darmstadt, Germany, 2012.

[2] Othonos, A., "Fiber Bragg gratings," Review of Scientific Instruments, 1997, vol. 68, no. 12, pp. 4309-4341.

[3] Froggatt, M., "Apparatus and Method for Measuring Strain in Bragg Gratings," U.S.Patent 5,798,521, 1998.

[4] Nicholas, M. J., Structural Analysis and Testing of a Carbon-Composite Wing Using Fiber Briggs Gratings, MS Thesis, 2013. 